\title{
Plasmid Encoding Antiangiogenic Metargidin Peptide
}

\author{
National Cancer Institute
}

\section{Source}

National Cancer Institute. Plasmid Encoding Antiangiogenic Metargidin Peptide. NCI

Thesaurus. Code C90537.

\begin{abstract}
A plasmid encoding the protein antiangiogenic metargidin plasmid (AMEP), the disinteg rin domain of ADAM-15 (metargidin), with potential antiang iog enic and antimetastatic activities. Upon intratumoral electrotransfer of plasmid encoding AMEP, AMEP binds to cellular integrin receptors alpha-v-beta-3 (avb3) and alpha-5-beta-1 (a5b1), which are upregulated on activated endothelial cells and a variety of tumor cells. Binding to the integ rin receptors may inhibit ang iogenesis and may inhibit tumor cell proliferation.
\end{abstract}

